\title{
La política local de seguridad en las grandes ciudades. El caso de la transformación de la policía municipal de Madrid
}

\author{
José Manuel RuANO DE LA FUENTE \\ Universidad Complutense de Madrid \\ jmruano@cps.ucm.es
}

Recibido: $01 / 11 / 2014$

Aceptado: 15/12/2014

\begin{abstract}
Resumen
En el año 2003 el gobierno de la ciudad de Madrid emprende una política integral de reforma de la policía local que tiene como objetivo colocar a la seguridad como servicio público central y actividad esencial de la policía frente a las tradicionales labores de vigilancia de edificios y regulación del tráfico. Sin embargo, el plan «Madrid Seguro» entra en conflicto con las inercias culturales de una organización tradicional y jerarquizada. La política de transformación de la policía actúa sobre varios ejes de intervención: cambios estructurales en la organización, del modelo de gestión, de la política de personal, incorporación de buenas prácticas externas, mejora de las infraestructuras y creación de alianzas.
\end{abstract}

Palabras clave: seguridad, policía local, reforma administrativa, gobierno local.

Security policy in big cities. The transformation of the local police in Madrid

\begin{abstract}
In 2003 the Government of Madrid implemented a comprehensive policy to reform the local police aiming to transform public safety as a core public service and as an essential activity of the police beyond the surveillance of official buildings and traffic regulation. However, the plan Madrid Seguro came into conflict with the cultural inertia of this traditional and hierarchical organisation. The reform scheme of the local police was based on several intervention areas: structural changes in the organisation design, the change of the management model, changes in the personnel policy, benchmarking, the improvement of infrastructures and the setting-up of partnerships with public and private institutions.
\end{abstract}

Key words: safety, local police, administrative reform, local governance.

\section{Introducción}

Las grandes ciudades se enfrentan a enormes desafíos en materia de seguridad. En la mayoría de las ciudades, las policías locales deben enfrentarse a contextos sociales especialmente desfavorables e institucionalmente complejos en los que la responsabilidad de la política de seguridad es compartida con niveles superiores de gobierno. Por otro lado, las políticas de seguridad más eficaces aprovechan las experiencias y cono- 
cimientos obtenidos en otros ámbitos para obtener mejores resultados. Así, no solamente es necesario contar con personal bien formado y con medios materiales modernos y suficientes, sino que cada vez hay mayor conciencia de la importancia que en materia de seguridad cobran factores como el liderazgo político, las políticas de inclusión social, la colaboración con la ciudadanía, el trabajo intergubernamental o el diseño urbanístico.

Siendo, por tanto, conscientes de que la política de seguridad en las grandes ciudades es el resultado de la combinación de variables diversas y de la interacción de distintas políticas, en el presente trabajo se pretende identificar los factores que están en la raíz del proceso de transformación de la policía local de Madrid iniciado en 2003 con el desembarco en el Ayuntamiento de Madrid del equipo encabezado por Alberto Ruiz-Gallardón procedente del gobierno de la Comunidad Autónoma. En el desarrollo del trabajo se ha utilizado la información estadística proporcionada por el Área de Gobierno de Seguridad y Movilidad del Ayuntamiento de Madrid correspondiente a los años 2003-2014, y la información cualitativa derivada de las entrevistas realizadas al Director General de Seguridad del Ayuntamiento y a la Jefe del Departamento de Evaluación y Calidad de los Servicios del Ayuntamiento de Madrid"

\section{La complejidad de las políticas urbanas de seguridad}

Las políticas de seguridad se han beneficiado en los últimos años de importantes avances tecnológicos que han permitido el diseño de sistemas de recogida y análisis de información y han logrado reducir los tiempos de respuesta. Son muchas las ciudades que han partido de este enfoque modernizador para mejorar la prestación de servicios, pero quizá el primer esfuerzo realizado en este sentido sea la utilización del sistema de geoprocesamiento COMPSTAT que se adoptó en la ciudad de Nueva York en los años 90 y que permitió a los mandos de la ciudad contar con un sistema de información integrado que incorporaba innovaciones en materia de gestión (Maguire, Mike and John, Tim, 2006: 71-74).

Por otro lado, los adelantos tecnológicos se han combinado en otras ciudades con estrategias de intervención orientada a la comunidad que, desde una óptica proactiva y mediante la colaboración con la ciudadanía, se centran ante todo en la prevención de los delitos. Este tipo de orientaciones se basa en la generación progresiva de vínculos de confianza con la población que permitan la discusión sobre la mejor manera de abordar los problemas de seguridad en el marco de instrumentos formales de cooperación (consejos vecinales de seguridad). Una variante de esta estrategia colaborativa es la empleada por la policía de proximidad británica o danesa, basada en la presencia regular de la policía en los barrios para transmitir sensación de seguridad a los vecinos

${ }^{1}$ El autor agradece las entrevistas concedidas para la redacción de este artículo a D. Pablo Escudero, Director General de Seguridad del Ayuntamiento de Madrid, y a Dña. Montserrat Rossell, Jefe del Departamento de Evaluación y Calidad de los Servicios. Las afirmaciones contenidas en el capítulo son responsabilidad exclusiva del autor. 
y establecer con ellos lazos de colaboración que les involucren en la solución de sus propios problemas mediante la adopción de posibles soluciones (Holmberg, Lars, 2002: 32-47). Otro ejemplo clásico de policía comunitaria es el sistema japonés koban, esto es, pequeños puestos de policía situados en las calles que permiten a la policía mantener relaciones a largo plazo con el público y recoger información en áreas conflictivas que no podría obtenerse de otro modo.

Ahora bien, debe reconocerse que ninguna de las estrategias y enfoques mencionados serían plenamente efectivos sin cambios culturales y sin transformaciones gerenciales en el manejo y análisis de la información. Debe tenerse en cuenta que la policía es la encargada de solucionar o atenuar al menos las consecuencias de muchos de los problemas sociales, económicos y políticos, y, en este sentido, los servicios de seguridad son simplemente una parte más de un sistema mucho más amplio. Así, el mantenimiento de relaciones cooperativas con otros actores institucionales y sociales a través de proyectos a largo plazo es un factor clave para el éxito de la política de seguridad.

Con todo, debe tenerse en cuenta que hay factores estructurales que condicionan la política de seguridad. Así, el diseño y la gestión del espacio público en las ciudades determinan la tasa de inseguridad y, en consecuencia, el tipo de estrategia policial más adecuada. Esta tesis sugiere que los ciudadanos contribuyen al orden y la seguridad solamente cuando se identifican con el espacio público y lo sienten como propio (Eck, John E.et al., 2005), y enlaza con otra de las teorías de mayor impacto práctico en la política de seguridad contemporánea: «la teoría de los cristales rotos». Desarrollada por Kelling y Wilson (1982), argumenta que el control de la delincuencia y la degradación del espacio público depende en buena medida del cuidado permanente de ese espacio por parte de las autoridades y de la represión de los pequeños comportamientos antisociales, enviando el mensaje al público de que esa área está vigilada y que la policía hace cumplir las normas. No obstante, suele reconocerse que esta estrategia logra sus mejores resultados en conjunción con técnicas basadas en sistemas de información, medidas de educación cívica y colaboración con la comunidad (Sampson, Robert J. y Raudenbush, Stephen W., 1999; Sousa, William H. y Kelling, George L., 2006).

Así pues, la cooperación con otras organizaciones públicas y con la comunidad son factores esenciales en la prevención y la lucha contra la delincuencia. No sólo es importante que los cuerpos policiales dispongan de adecuados y modernos sistemas de información, sino que sean capaces de compartirla para alcanzar una mayor eficacia. En ese sentido, la evaluación de la acción pública es un desafío de extraordinaria magnitud toda vez que debe estar fundada en criterios pertinentes que den cuenta de las preocupaciones de la comunidad, la información debe estar disponible y debe ser útil y fiable (es más fácil saber cuántos sospechosos han sido arrestados que cuántos delitos se han prevenido), y debe considerar en última instancia el nivel de confianza ciudadana en la acción de la policía. Por todo ello, es necesario desarrollar técnicas de evaluación apropiadas al contexto administrativo y tecnológico en el que opera la organización. Pero, además, la creación de alianzas público-privadas en el ámbito de la seguridad (con asociaciones de comerciantes, de vecinos o escuelas) ha sido un factor de primer orden en el diseño de políticas preventivas. Este enfoque reticular exige tejer relaciones basadas 
en la confianza mutua entre actores públicos y privados para crear al cabo las condiciones óptimas necesarias que garanticen un nivel de eficacia aceptable.

Por último, otro factor que puede inclinar la balanza de la acción policial hacia el éxito o el fracaso es el contexto institucional en el que opera la policía local. En las iniciativas de reforma de las políticas de seguridad tiene un peso fundamental la determinación del rol que corresponde desempeñar a cada cuerpo policial (nacional, regional o local) en contextos políticos descentralizados y la puesta en marcha de iniciativa de colaboración entre organizaciones pertenecientes a niveles de gobiernos diferenciados. Del mismo modo, la relación entre los responsables políticos y el resto de personal es importante: si uno de los dos elementos de la organización (los líderes políticos o el personal funcionario) adoptan una postura de resistencia al cambio, la reforma se topará con obstáculos adicionales.

Vistos los factores influyentes en la política de seguridad, se analiza a continuación el contexto del proceso de reforma de la policía local madrileña, los ejes de intervención de la política de reforma y sus resultados.

\section{El contexto de la reforma de la policía local de Madrid}

La creación de la policía municipal de Madrid se remonta a 1838, año en que se aprueba su primer reglamento orgánico. La llegada de la democracia y la aprobación de la Constitución de 1978 supusieron un gran cambio del cuerpo policial tanto en su concepción como en su funcionamiento al ajustarse a los principios y normas del Estado de Derecho. Más adelante, en los últimos años del siglo XX aumentan notablemente sus recursos materiales y humanos hasta configurarse como una de las estructuras policiales más numerosas de España.

En los últimos años, el plan estratégico «Madrid Seguro» propició un cambio profundo que afecta a las formas de gestión, las infraestructuras físicas y la concepción global de un modelo policial que pasa a considerarse como un servicio atento a las transformaciones sociales y al servicio de la ciudadanía. Como se verá más adelante, estos parámetros ideológicos entran en contradicción con las fuertes inercias culturales de una organización centenaria, muy jerarquizada, excesivamente burocratizada por la vigencia de una normativa muy rígida, con una alta sindicalización y una pertinaz carencia de recursos materiales y humanos.

Coincidiendo con el cambio de siglo y los cambios que le acompañan (sociales, culturales, demográficos, tecnológicos, globalización, internacionalización y diversificación de la delincuencia), se impuso la necesidad de iniciar un proceso de transformación de la policía de Madrid para hacer de ella una policía «integral» que diese preferencia a la seguridad como servicio público central, aun a costa de la asunción de nuevas competencias, frente a la tradicional preponderancia de la regulación del tráfico y la custodia de edificios públicos.

En este cambio de orientación desempeñó un papel esencial el cambio de gobierno encabezado por Alberto Ruiz-Gallardón en mayo de 2003 y la consiguiente llegada al Ayuntamiento de Madrid de un equipo con experiencia previa en políticas de reforma administrativa procedente de la Comunidad Autónoma de Madrid. La puesta en marcha en el mismo año 2003 del plan «Madrid Seguro» partía de la ampliación del 
ángulo de visión de la política de seguridad a partir de la premisa de que ésta es la base de cualquier actividad, de que todos los servicios y actividades sociales, con independencia de su naturaleza (cultural, empresarial, medioambiental, urbanística,...) requieren de una política de seguridad efectiva.

Además, este papel central concedido a la seguridad se vio respaldado por dos argumentos coadyuvantes: por un lado, la constatación de que la gran mayoría de delitos y faltas denunciados en la ciudad de Madrid son de competencia municipal (tráfico, venta ambulante, ruidos, ...); y, por otro, que los sucesivas encuestas aplicadas a los ciudadanos reflejan una demanda mayoritaria y constante de más policía municipal en la calle como factor de prevención y disuasión, lo que aumenta al tiempo la sensación de seguridad subjetiva (Greneron, 2010: 136). En este sentido, el «barómetro de consumo» de 2003 ya reflejaba la preferencia ciudadana de que los policías se dedicasen fundamentalmente a labores de seguridad frente a otras tareas consideradas secundarias. Por último, la integración de la seguridad en el debate público y la discusión sobre el papel de las policías municipales en las grandes ciudades terminarán por impulsar el proceso de reforma.

La transformación, por lo tanto, de la concepción de la policía madrileña se inició gracias al cambio del equipo de gobierno y, así, el plan «Madrid Seguro» tendrá su continuidad garantizada en las sucesivas ediciones de los períodos 2004-2007, 20082011 y 2012-2015, tras las sucesivas victorias electorales del PP y el mantenimiento de la política de reforma. Sin embargo, este proceso de cambio tendrá que hacer frente a numerosas resistencias que se manifestaban en la desconfianza de sectores del poder judicial y de la policía nacional frente al proyecto de «policía integral», que implicaría su conversión en policía judicial; su escasa capacidad organizativa derivada de la existencia de instrumentos de selección y promoción del personal rígidos y obsoletos combinados con una alta participación sindical; la ausencia de sistemas que vinculasen la dirección a objetivos de gestión; la alta conflictividad sindical; una baja productividad; y un alto índice de absentismo laboral.

\section{Los ejes de la política de reforma}

Estos grandes desafíos a los que debía hacer frente la política de reforma fueron abordados desde el diseño de varios ejes de actuación simultáneos que buscaban su coherencia en la premisa de que la seguridad debía ser una política clave en la acción de gobierno municipal y que debía estar orientada a las demandas de los ciudadanos y a la obtención de resultados mensurables.

\subsection{El cambio en la estructura organizativa}

Uno de los factores básicos del proceso de impulso de la estrategia de cambio en la policía fue la reestructuración organizativa. Los servicios de seguridad se encuadraron en el Área de Gobierno de Seguridad y Movilidad, que pasó a subdividirse en dos 
Coordinaciones Generales: Seguridad y Emergencias, donde se encuadra la policía, los servicios de emergencias y protección civil; y Movilidad, que se ocupa de todo lo referente a la organización del transporte público en la ciudad. Cada uno de los 21 distritos que conforman la ciudad de Madrid dispone de una Unidad de Policía Municipal al mando de un oficial y cuenta con un grupo de agentes tutores, especializados en la protección a la infancia y la vigilancia de los entornos escolares, y con Oficinas de Atención al Ciudadano, que recogen y atienden las demandas de los ciudadanos en todo lo que se refiere a la convivencia vecinal.

El despliegue de la policía en los distritos y los barrios pretende, por una parte, incidir en la política de prevención frente al carácter tradicionalmente reactivo de los servicios policiales $\mathrm{y}$, por otra, buscar generar cauces de colaboración con vecinos y comerciantes en espacios de generación y expresión de demandas ciudadanas en materia de seguridad: las Juntas Locales de Seguridad y las Asociaciones de vecinos, de padres y madres, comerciantes e inmigrantes.

La reorganización de la estructura orgánica de la policía persigue en definitiva centrar la mayor parte del esfuerzo en las tareas de seguridad. Para ello, se crea como órgano de gestión la Dirección General de Seguridad, cuyos nuevos miembros civiles sustituyen a los mandos policiales y profesionalizan las tareas de apoyo a la gestión policial: los «corbatas», denominados así despectivamente por los mandos policiales tradicionales, asumen por vez primera las funciones de dirección. Todo un símbolo de los nuevos tiempos. Además, se crean las Unidades Centrales de Seguridad, órganos especializados en labores de seguridad sobre el terreno («top manta», inspección de locales de ocio, menudeo de droga, etc.); y la externalización generalizada de las labores de vigilancia en edificios públicos, junto con la creación del Cuerpo de Agentes de Movilidad, permite liberar a la policía de tareas que añaden poco valor a la política de seguridad (tareas administrativas, vigilancia de edificios y control estático del tráfico).

\subsection{El cambio en el modelo de gestión.}

La policía de Madrid comienza a orientar su gestión hacia el logro de resultados a partir de la satisfacción de las expectativas y necesidades de los ciudadanos para lograr un impacto positivo en la sociedad. Esta filosofía de actuación, que coincide con la línea de modernización administrativa y de mejora de la gestión emprendida por la Coordinación General de Seguridad, se traduce en la apuesta decidida por la evaluación de resultados y la detección de áreas de mejora según el modelo de excelencia EFQM (European Foundation for Quality Management), que es utilizado en Europa como un modelo de referencia desde 1988 (Arenilla, 2011). Mediante su uso, se pretende por vez primera integrar en la organización policial la cultura de la calidad organizativa mediante el uso de un modelo de gestión que permite reflexionar sobre los objetivos de la organización y los procedimientos y planes de mejora. Así, la Coordinación General de Seguridad obtuvo su primera certificación de calidad EFQM a finales de 2006, lo que la convirtió en el primer servicio municipal del Ayuntamiento de Madrid, y en la primera policía de España y del resto de las capitales europeas en obtener este reconocimiento. En 2008 obtuvo el sello europeo de calidad EFQM en su cate- 
goría de más de 400 puntos. El resultado práctico fue la evaluación generalizada de las prácticas de gestión y el análisis de todas las actividades de la organización. En diciembre de 2010 se obtuvo un nuevo reconocimiento a la nueva orientación gerencialista de la policía mediante el logro del sello europeo de calidad EFQM de más de 500 puntos.

El empleo de la dirección por objetivos (DPO) desde 2005 se generalizó mediante la asunción creciente indicadores y la ampliación de su alcance a la escala ejecutiva y a la Dirección General de Seguridad. Se pasó de la utilización de 18 indicadores en 2005 a 107 indicadores estables para el período 2008-2011 que son consensuados con los responsables de las unidades. En la actualidad, los indicadores de la DPO están constituidos por los indicadores de valor público y los indicadores que miden los compromisos adquiridos con la ciudadanía en su carta de servicios. Las unidades del cuerpo analizaron su actividad policial para tratar de identificar las mejores prácticas y compartirlas con el resto de unidades. La meta era, en la medida de lo posible, estandarizar y homogeneizar formas de trabajo, mejorar la coordinación entre unidades, definir objetivos, y utilizar de herramientas de seguimiento y de corrección de objetivos y actividades en un contexto cultural de mejora continua.

En la misma línea de mejora, se incidió en el Modelo de Gestión por Competencias para la dirección de los recursos humanos de la organización. Se entendía que la capacitación de los agentes del cuerpo exige una permanente actualización al asumir nuevas funciones y entrar en nuevas áreas normativas. La elaboración de un plan de formación eficaz requería una detección previa de necesidades, por lo que se procedió a definir y describir los puestos de trabajo de la totalidad de la plantilla por categorías profesionales, $\mathrm{y}$ a determinar los perfiles de competencias correspondientes. Para cada una de las 18 competencias finalmente definidas, se fijaron seis niveles de desempeño que pasaron a ser factores de evaluación del rendimiento individual.

El sistema de gestión de procesos puesto en marcha supuso una radical transformación del modelo de gestión tradicional, que descuidaba los objetivos de la actuación del servicio mediante el empleo de indicadores de gestión, el cultivo de las relaciones con la ciudadanía, y especialmente con los grupos de ciudadanos y de presión más concernidos directamente, y la detección de áreas de mejora en la gestión. A partir de cada línea estratégica de actuación, se relacionó cada objetivo con los indicadores que permitían evaluar su implantación efectiva. La dirección de la policía identificó las oportunidades de mejora en los procesos de prestación de los servicios a partir de un proceso de planificación capaz de prever situaciones de riesgo y aportar la información necesaria para mejorar la prestación de los servicios procedente de las quejas, reclamaciones, análisis de la percepción de los ciudadanos, seguimiento de los resultados y la misma experiencia de los agentes. A este sistema de planificación contribuía el diseño de un mapa de procesos para toda la Coordinación de Seguridad en el que se definían las líneas de actuación, sus costes y su grado de estandarización.

Esta metodología se comenzó a aplicar de forma sistemática a partir de toda la información recabada y analizada procedente tanto de fuentes internas como externas a la organización. Entre estas últimas, los mecanismos de detección y análisis de demandas, expectativas y necesidades de los ciudadanos desempeñaban un papel central, y terminaron incidiendo en el diseño y en el desarrollo de los servicios, e incluso en la concepción misma de la actuación de la policía, que pasa de prestar «servicios 
policiales» a ofrecer un «servicio integral de seguridad» capaz de adaptarse a las expectativas de los ciudadanos y de anticiparse a sus necesidades futuras en función del tipo de «ciudadano-cliente». Esta perspectiva, que persigue la segmentación del ciudadano según su posición ante los servicios de seguridad y que modula la intervención de la policía en función de esa posición, refleja la preocupación por la atención prestada al ciudadano, al que hay que tratar de la misma forma que lo hace una organización privada en un contexto de competencia de mercado, y que es capaz de exigir y ejercer sus derechos no sólo como ciudadano sino como usuario-cliente del servicio considerado (García Solana, 2001). Y para captar esas necesidades, es imprescindible, como se verá más adelante, tener presencia cercana a los lugares de encuentro con los ciudadanos: foros en los que se discuten las demandas de los ciudadanos y los problemas de los distritos (las Juntas Locales de Seguridad), las asociaciones de vecinos, los diversos canales de acceso al sistema de sugerencias y reclamaciones del Ayuntamiento de Madrid (presencial, telefónico e informático), las oficinas de atención al ciudadano, el barómetro de seguridad y la encuesta de calidad de vida en la ciudad, al que se han incorporado preguntas sobre seguridad que son también una fuente para la detección de las necesidades y expectativas de los distintos «clientes» de la policía.

Un reflejo del empleo de nuevos instrumentos de mejora orientados a la ciudadanía es la utilización de Cartas de Servicio con finalidad informativa sobre el grado de compromiso con los niveles de calidad comprometidos y como factor de aprendizaje organizativo derivado de su aplicación y evaluación (Crespo y Criado, 2005; Arenilla, 2011). En efecto, la aprobación de la «Carta de Servicio de Atención al Menor» en 2006 y la «Carta de Servicios de Atención a la Ciudadanía de la Policía Municipal» en junio de 2008, que por vez primera permite incorporar en la dirección por objetivos los compromisos de servicio adquiridos en la Carta, supusieron la culminación del trabajo de análisis de los procesos de atención al ciudadano en la aplicación de una herramienta que persigue la doble finalidad de informar a la ciudadanía sobre los compromisos y servicios asumidos por la policía, y de fijar unos objetivos de calidad y unos indicadores que permitan hacer el seguimiento de aquellos. En esta labor, la policía trabaja con distintos perfiles de ciudadanos que cuentan con expectativas de trato y de servicio diferenciados según su posición ante la policía (como víctima, delincuente, infractor, testigo, afectado, etc.).

\subsection{La incorporación de buenas prácticas externas}

En el proceso de cambio de la cultura organizativa de la policía madrileña es esencial el conocimiento y la incorporación de las mejores prácticas de organizaciones equivalentes externas (benchmarking) para poder responder eficazmente a las necesidades sociales y poder abordar las formas más novedosas de delincuencia.

Así, la policía municipal de Madrid tomó de la policía de Nueva York los procesos de gestión del cambio y la orientación de los servicios a los resultados. En un contexto de ciudadanía insatisfecha con los servicios de seguridad, unos agentes desmotivados, con pocos estímulos profesionales y la anteposición del esfuerzo (el número de patrullas) sobre los resultados (el descenso de la criminalidad), el alcalde Rudolph Giulani inició un proceso de renovación tecnológica y metodológica que se tradujo en la 
presencia de más agentes en las calles de la ciudad norteamericana, en la sustitución de los incentivos basados en las horas extras por el establecimiento de recompensas en función del rendimiento, y en la puesta en práctica de un nuevo modelo de reparto de responsabilidades de alta dirección entre una estructura de gestión a cargo de personal civil designado por el alcalde y una estructura operativa dirigida por personal uniformado.

El segundo de los referentes directos de la policía de Madrid fue la Policía Metropolitana de Londres, de la que tomó la gestión diferenciada de los procesos operativos y los de soporte, y especialmente el concepto de policía al servicio de la comunidad. Desde hace años, la policía londinense desarrolla el programa de «policía comunitaria», prestado por «agentes de apoyo a la comunidad». Este programa, originalmente aplicado en Ámsterdam por agentes colaboradores que no pertenecen al cuerpo de policía en barrios de alta presencia de población inmigrante, consiste en el despliegue en los barrios de agentes de la misma etnia u origen racial de la mayoría de los vecinos para tratar de conseguir una mejor aceptación de su presencia y crear canales de información para la elaboración de planes de prevención y de intervención. La exploración y generación de alianzas de la policía madrileña con los representantes vecinales, asociaciones y comerciantes tiene su origen en las experiencias de la policía comunitaria inglesa.

\subsection{El cambio en la política de personal}

La estrategia de personal es quizá el factor protagónico y más complejo del proceso de cambio. En primer lugar, porque abarca aspectos muy diversos que deben coordinarse adecuadamente: la creación y dotación presupuestaria de la Dirección General de Seguridad, el número total de personal, la relación entre el personal en calle y en oficina, la regulación de los turnos, la cobertura de vacantes, los procesos de selección y promoción, la política retributiva, la dotación de medios materiales, los sistemas de formación y los mecanismos de evaluación del rendimiento. La transformación en materia de personal se abordó de dos maneras:

La primera, a través de un incremento y rejuvenecimiento de la plantilla que abarca el período 2003-2008. Este fuerte crecimiento de la plantilla se tradujo en un aumento de más de 1.000 policías y en 300 efectivos no uniformados en tan sólo cinco años, entre los que se encuentra el personal administrativo, cuyo crecimiento permitió liberar a los policías de las tareas burocráticas y tener más presencia en la calle. De este modo, se pasó de 2.300 policías que prestaban servicio en los distritos en 2003 a 5.300 policías dedicados a seguridad ciudadana, de un total de casi 6.400 policías en plantilla en 2013. Este cambio en las funciones del personal uniformado fue también posible gracias a la creación del cuerpo de agentes de movilidad, que asumió la regulación del tráfico, y al abandono de las tareas de gestión y administración, absorbidas por la Dirección General de Seguridad, así como de las labores de vigilancia de edificios públicos, contratadas con empresas privadas de seguridad. Estas medidas permitieron la liberación de unos 1.500 agentes para aumentar la dotación en los distritos, la creación de 174 agentes tutores dedicados exclusivamente a la seguridad en el entorno 
escolar, el despliegue de una oficina de atención del ciudadano en cada uno de los 21 distritos, que cuentan además con una oficina móvil, y la creación de dos unidades centrales de seguridad que sirven apoyo los fines de semana a las unidades de distrito.

Figura 1. Evolución de la plantilla de la policía municipal

\begin{tabular}{|c|c|c|c|}
\hline Año & Personal uniformado & Otras categorías & Total \\
\hline 2002 & 5663 & 106 & 5.769 \\
\hline 2013 & 6455 & 336 & 6.791 \\
\hline
\end{tabular}

Fuente: Área de Gobierno de Seguridad y Movilidad 2013.

El resultado final ha sido el aumento de la ratio de policía por cada 1.000 habitantes hasta los 2,1 en 2013, superando en más del doble la ratio de un agente por 1.000 habitantes recomendada por la Unión Europea, con lo que la plantilla del personal asignado a los servicios de seguridad vendría a equivaler a la tercera parte de todo el personal del Ayuntamiento de Madrid, lo que da idea de la prioridad otorgada a los servicios de seguridad por parte del gobierno municipal. Naturalmente, la apuesta por la política de seguridad tiene su reflejo en el crecimiento del presupuesto ejecutado por la policía, que pasa de 249 millones de euros en 2003 a los 329 millones en 2012 (un $32,2 \%$ de aumento), y en el que el gasto en personal consume la mayor parte del presupuesto $(95,8 \%)$.

Figura 2. Evolución del presupuesto de la policía municipal (en millones de euros)

\begin{tabular}{|l|r|r|r|r|r|r|r|r|r|r|}
\hline \multicolumn{1}{|c|}{ Concepto } & $\mathbf{2 0 0 3}$ & $\mathbf{2 0 0 4}$ & $\mathbf{2 0 0 5}$ & $\mathbf{2 0 0 6}$ & $\mathbf{2 0 0 7}$ & $\mathbf{2 0 0 8}$ & $\mathbf{2 0 0 9}$ & $\mathbf{2 0 1 0}$ & $\mathbf{2 0 1 1}$ & $\mathbf{2 0 1 2}$ \\
\hline TOTAL & 249,0 & 296,0 & 336,5 & 368,9 & 380,7 & 393,7 & 390,1 & 364,6 & 372,1 & 328,7 \\
\hline Personal & 237,5 & 279,6 & 309,9 & 339,2 & 358,9 & 377,2 & 381,1 & 351,9 & 358,4 & 317,6 \\
\hline Gasto corriente & 7,1 & 8,2 & 10,6 & 13,6 & 10,5 & 13,2 & 13,2 & 11,3 & 13,4 & 10,7 \\
\hline Inversiones & 4,4 & 8,2 & 16,0 & 16,1 & 11,3 & 3,3 & 0 & 1,4 & 0,3 & 0,4 \\
\hline
\end{tabular}

Fuente: adaptado de Policía Municipal de Madrid (2003-2012)

Y la segunda gran transformación se produjo en el período 2010-2014. Se centró en la adecuación de la plantilla a las necesidades de la ciudad, incrementando el número de patrullas en los turnos de tarde y noche y en fines de semana. El incremento de la patrullas se consiguió mediante la modificación de los cuadrantes de trabajo de la noche y la inclusión dentro de las jornadas laborales de 4 jornadas adicionales en sábado y domingo a través de la firma de un nuevo acuerdo de condiciones laborales suscrito por todas las centrales sindicales excepto UGT, que no tiene apenas representación en policía. 
Figura 3. Media de patrullas en fin de semana (viernes a domingo)

\begin{tabular}{|c|c|c|c|c|c|}
\hline Año & $\mathbf{2 0 1 0}$ & $\mathbf{2 0 1 1}$ & $\mathbf{2 0 1 2}$ & $\mathbf{2 0 1 3}$ & $\mathbf{2 0 1 4}$ \\
\hline Media patrullas & 1.762 & 1.906 & 1.834 & 2.005 & 2.287 \\
\hline \% incremento s/2010 & & $\mathbf{8 , 1 3}$ & $\mathbf{4 , 0 9}$ & $\mathbf{1 3 , 7 6}$ & $\mathbf{2 9 , 7 8}$ \\
\hline
\end{tabular}

Fuente: Área de Gobierno de Seguridad y Movilidad 2010-2014.

Más allá del análisis de los datos cuantitativos, la separación en la Coordinación General de Seguridad de la parte operativa de la parte de dirección permitió profesionalizar la gestión de toda la organización y facilitó la introducción de formas de gestión del personal habituales en el mundo privado, pero menos conocidas y generadoras de tensiones en las organizaciones públicas. Es el caso de la aplicación de la dirección por objetivos, a la que ya se hizo referencia, y de los cambios en los sistemas de retribución basados en el rendimiento individual para dar cauce a la iniciativa, ambición e implicación personales.

Así, uno de los focos más fuertes de resistencia al cambio se localiza en los representantes del cuerpo de policía. Con una alta sindicalización de carácter gremial, que se acerca al $80 \%$ del total de la policía, cualquier propuesta de alteración de sus condiciones de trabajo ha sido recibida con oposición de distinta intensidad. Configurada hasta 2003 como una organización muy jerarquizada, la policía de Madrid funcionaba como una burbuja aislada que disponía de reglas y normas propias dentro del Ayuntamiento de Madrid. Las primeras muestras de resistencia se debieron a la pérdida de poder de los mandos uniformados, a la introducción de indicadores que guiasen la actuación policial y a la centralidad de la opinión de los ciudadanos, considerada como algo cambiante a lo que no merecía la pena prestar excesiva atención. Aun hoy, las nuevas autoridades no han logrado vencer todos los obstáculos a la flexibilización de la gestión de los recursos humanos. Las resistencias siguen siendo fuertes en materia de movilidad del personal, diseño de relaciones de puestos de trabajo y competencias, a la introducción de nuevos instrumentos de acceso (como las entrevistas personales o la evaluación de la práctica profesional) y de promoción del personal, y, por supuesto, a la aplicación de incentivos salariales en función del rendimiento.

Así, en la primavera de 2010 el proyecto de reparto de seis millones de euros en función de la productividad (medida como jornadas trabajadas) y de la consecución de objetivos (reducción de la tasa de criminalidad, del número de víctimas mortales en accidentes de tráfico, de los tiempos de respuesta y de la delincuencia dirigida a turistas) se encontró con la oposición frontal de los dos grandes sindicatos policiales, que solicitaban un reparto lineal de los fondos y la contratación de más personal. La oposición a la introducción de estos incentivos retributivos dificulta sin duda la lucha contra el alto absentismo (de 29 días al año de media en 2009) para las 193 jornadas laborales anuales de un policía madrileño. Estas políticas de recursos humanos se han traducido, finalmente, en una reducción del absentismo, como puede verse en la tabla siguiente. 
Figura 4. Media de días de baja por trabajador (2009-2013)

\begin{tabular}{|l|l|}
\hline 2009 & 29,0 \\
\hline 2010 & 25,2 \\
\hline 2011 & 24,2 \\
\hline 2012 & 21,5 \\
\hline 2013 & 20,6 \\
\hline
\end{tabular}

Fuente: Área de Gobierno de Seguridad y Movilidad 2009-2013.

\subsection{El cambio en las infraestructuras}

También el capítulo de inversiones experimentó un fuerte aumento desde 2003 (ver figura 2), especialmente durante los tres primeros ejercicios. Se pasa de 4,4 millones de euros en 2003 a más de 16 millones en 2006. Esta inversión inicial va reduciéndose en los años siguientes. Así, en 2007, se invierten 11,3 millones, de los que 7,5 se dedican en ese año al Centro Integral de Seguridad y Emergencias de Madrid (CISEM), considerado un proyecto estratégico.

Con la finalidad de dar mayor visibilidad y accesibilidad a la policía municipal, se acometió un plan de renovación de 31 de los 40 edificios y oficinas de la Coordinación General de Seguridad con un coste de 10,5 millones de euros en tres años. Además, mediante leasing se renueva y se ajusta la flota de vehículos a las necesidades y características del servicio, de modo que en la actualidad la flota de policía alcanza los 500 coches y las 500 motos adaptados con la incorporación de elementos de seguridad, como puertas blindadas, dispositivos de reconocimiento óptico para la detección de infracciones y localización automática de vehículos robados, GPS, ordenadores y radios. A todo este esfuerzo inversor, se añadió la renovación total del vestuario, ahora diseñado para el trabajo en la calle y no tanto en oficina. Es evidente que se ha dado un salto cualitativo en la modernización de las infraestructuras y las tecnologías de información y de comunicación para tratar de situar a la policía madrileña como organización de referencia en su sector a nivel nacional e internacional. Se pone de manifiesto que la renovación tecnológica es una de las claves del plan de modernización de la policía madrileña para la optimización del rendimiento de gestión y la reducción de los tiempos de respuesta a las demandas ciudadanas.

Madrid cuenta con más de 10.000 profesionales dedicados a la prestación de servicios de seguridad y emergencias las 24 horas al día durante todo el año, de los cuales unos 2.000 deben coordinarse en su trabajo diario. En este contexto, el CISEM fue concebido como uno de los proyectos más ambiciosos del Área de Gobierno y Seguridad y uno de los programas tecnológicos más avanzados de todo el Ayuntamiento. La inversión en la creación del CISEM fue de unos 21 millones de euros y está dotado de infraestructuras de comunicación de última generación, salas de control de tecnología avanzada y aplicaciones informáticas integradas para la configuración de un sistema 
pionero en la coordinación y gestión de la seguridad y emergencias de una gran ciudad. Su principal virtualidad consiste en que permite agrupar a todos los servicios de emergencias de la ciudad (policía, SAMUR, protección civil, agentes de movilidad y bomberos) a partir de la misma base tecnológica para que puedan compartir información y afrontar de este modo las incidencias de forma sinérgica y más eficientemente. Este centro integral de coordinación de los servicios de seguridad y emergencias emerge como el eje principal de operaciones de gestión de crisis. Los sistemas informáticos permiten trabajar con un incidente de forma única («sistemas de incidente único») con independencia de los cuerpos que participen en su resolución. El centro está auxiliado además por la red privada de comunicaciones TETRA, que garantiza un sistema alternativo de comunicación de forma permanente. La apuesta estratégica por la gestión centralizada y por la coordinación de todos los servicios de seguridad y de emergencias fue posible gracias a una decisión política previa: la conformación de una única área de gobierno bajo la autoridad de un mismo concejal. Se confirma así que ciertos programas transversales requieren de contextos administrativos homogéneos en los que dirección política facilita la coordinación operativa (Knoepfel, Larrue y Varone, 2001: 200-211).

\subsection{La creación de alianzas}

Ya se ha hecho referencia a que una las acciones estratégicas de la policía madrileña ha consistido en tratar de generar alianzas con otras organizaciones, tanto dentro como fuera del Ayuntamiento de Madrid (administraciones, representantes de colectivos ciudadanos, asociaciones) para implicar tanto a unas como a otras en la construcción de un concepto de seguridad que contribuya al aumento de la calidad de vida y, en esta empresa, coopere con sectores (urbanismo, educación, servicios sociales, limpieza) aparentemente ajenos.

De este modo, la policía municipal colabora de forma general, en el ámbito del Ayuntamiento de Madrid, con las Juntas Municipales de cada Distrito (a través de las unidades integrales de distrito y, directamente, mediante comunicación entre el concejal del distrito y el jefe de la unidad de policía); y con diferentes servicios centrales: medio ambiente, informática, patrimonio, obras, y familia e infancia, entre otros ${ }^{2}$.

Asimismo, resulta indispensable la creación de alianzas con el resto de los «actores de la seguridad» en la ciudad de Madrid: la Delegación del Gobierno, la Guardia Civil y la Fiscalía. Estas actividades de colaboración se han materializado en la firma de convenios y protocolos de actuación conjunta en materia de emergencias, aduanas, tráfico y protección a la naturaleza. Por otra parte, el objetivo de la transformación de la policía municipal en una policía «integral» pasa por su conversión en policía judi-

2 En colaboración con la Concejalía de Empleo, se puso en marcha el «Plan Japón», proyecto que pretendía mejorar la negativa percepción de la seguridad en la ciudad de Madrid entre los turistas japoneses, lo que había provocado un descenso de sus visitas a la capital. 
cial, por lo que la adscripción de la policía municipal de Madrid al Poder Judicial para la realización de funciones de policía judicial ha sido una iniciativa pionera que ha terminado trasladándose a otros partidos judiciales de la Comunidad de Madrid y a otras partes de España. En esta misma línea, los acuerdos firmados con distintos organismos judiciales ${ }^{3}$ permiten el establecimiento de cauces de comunicación y actuación en materia de siniestralidad laboral y la investigación de accidentes laborales y redacción de atestados para la autoridad judicial. Además, la firma de un convenio con el Ministerio del Interior ha permitido la participación de la policía municipal en el diseño y desarrollo de las políticas de seguridad ciudadana, y la puesta en marcha de mecanismos que facilitan la coordinación entre la policía local y los cuerpos de seguridad del Estado.

Así pues, en una primera fase, la firma del protocolo de actuación de 6 de septiembre de 2006 permitió la intervención de la policía municipal de Madrid en temas de siniestralidad laboral; y un segundo protocolo, firmado el 27 de marzo de 2007, le otorgó competencias de policía judicial en una serie de delitos menores y faltas, aumentando de esta forma su campo de actividad en distintos ámbitos que se suman a las funciones históricamente asumidas en materia de tráfico. Este nuevo papel permite a los agentes municipales acudir inmediatamente al lugar de los hechos, realizar el atestado y llevar a cabo las investigaciones para el poder judicial. La legislación vigente (Ley 2/1986 de Fuerzas y Cuerpos de Seguridad del Estado), al circunscribir las competencias de policía judicial a la Policía Nacional y a la Guardia Civil, y al reducir el papel de las policías municipales a mero actor colaborador de éstas, deja de lado a policías locales que, por su capacidad operativa y formación, pueden ser una herramienta más de investigación, pueden acortar los plazos de los procedimientos $\mathrm{y}$, en definitiva, pueden contribuir a dar una respuesta coordinada a los desafíos en materia de seguridad. Este es el caso de la policía municipal de Madrid, que, mediante la firma de estos acuerdos de colaboración con otros actores, ha conseguido transformarse en una policía integral al tiempo que se ha dado respuesta con ellos a las insuficiencias de una legislación anticuada para dar respuesta a los retos que plantea la seguridad como competencia compartida.

Sin embargo, como ya ha quedado de manifiesto, los principales «clientes» en el marco del plan de reforma de la policía madrileña son los ciudadanos y, por esta razón, es a ellos a quienes se dirigen buena parte de los esfuerzos para la generación de alianzas. Mediante la presencia policial en las calles y al acercamiento a los representantes vecinales se ha acentuado la apuesta por la prevención frente a la reacción. Son evidencias de esta preocupación el incremento de la dotación de personal de la Unidad de Agentes Tutores, que pasa de 36 agentes en 2003 a más de 150 en la actualidad, y que están dedicados a la seguridad escolar en coordinación con las asociaciones de vecinos y las asociaciones de padres y madres de estudiantes; la creación, con 82 agentes, de

${ }^{3}$ Hay acuerdos firmados con el Fiscal Jefe de Madrid y el Juez Decano, y un convenio con la Fiscalía del Tribunal Superior de Justicia de la Comunidad de Madrid y el Decano de los Juzgados de Primera Instancia e Instrucción. Cabe añadir entre los actores estratégicos a los proveedores tecnológicos para el suministro de medios materiales e informáticos. 
la Unidad de Atención y Protección a la Familia, dedicada a la prevención de la violencia doméstica; y la creación de la Unidad de Apoyo y Coordinación con la Comunidad Educativa, que cuenta con 87 policías para dar clase a los escolares sobre seguridad vial.

\section{Los resultados de la política de reforma de la policía municipal}

Una vez iniciada la política de reforma en 2003, a partir de la llegada al gobierno municipal del equipo encabezado por el alcalde Ruiz-Gallardón, la policía municipal adoptó progresivamente el cambio en el modelo de organización y una nueva misión impulsada desde el ámbito político: «prestar un servicio público de seguridad que garantice una ciudad segura y un vecino protegido, basado en los principios de prevención, cercanía, participación ciudadana y solidaridad $\gg^{4}$. Esta misión, llevada a la práctica a través de los procesos de reforma que han sido descritos, ha dado resultados positivos a lo largo de los últimos años.

\section{Figura 5. Índice de delitos y faltas por 1.000 habitantes}

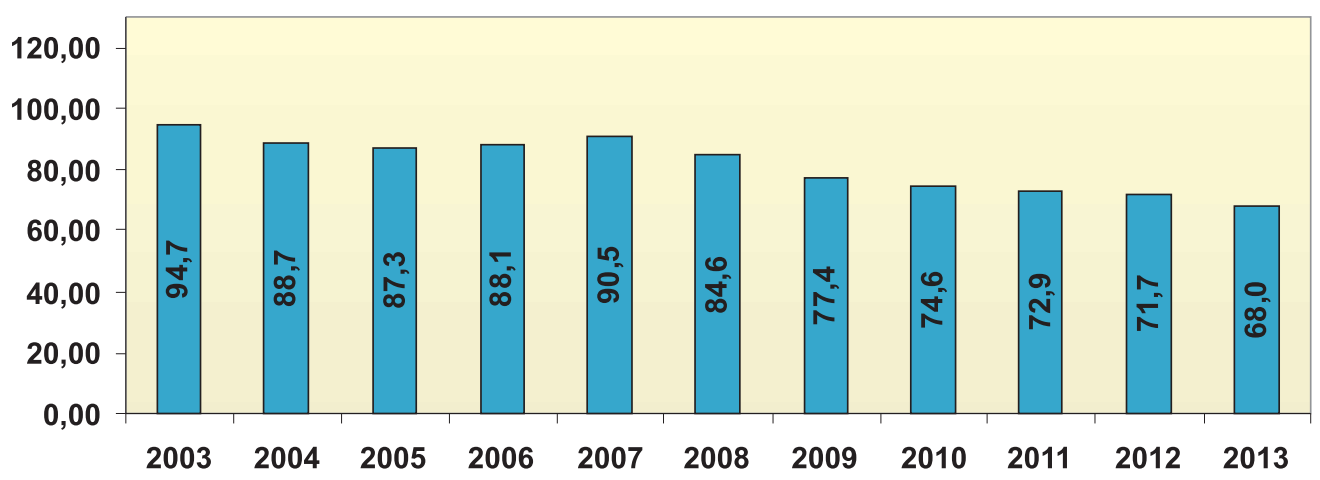

Fuente: Área de Gobierno de Seguridad y Movilidad (2003-2013)

Por lo referido a la seguridad objetiva, se ha pasado de una tasa de criminalidad, incluyendo los delitos y faltas cometidos por mil habitantes, de 94,72 en 2003, a una tasa de 68 en 2013. Como puede verse en la figura 5, la tendencia a lo largo de estos seis años es claramente positiva, a pesar de que la población de la ciudad de Madrid pasa de los 3.092.759 habitantes de 2003 a los 3.215.633 de 2013.

\footnotetext{
${ }^{4}$ Misión de la policía municipal según el Plan Estratégico «Madrid Seguro».
} 
Por otra parte, el Eurobarómetro sitúa a Madrid entre las ciudades más seguras de Europa, el número de víctimas mortales en accidentes de tráfico ha pasado de 78 en 2003 a 32 en 2013; y el aumento de la eficacia del servicio policial ha pasado del 5,9 en 2005 al 6,5 en 2008 (ver figura 6).

Figura 6. Evolución del índice de eficacia policial

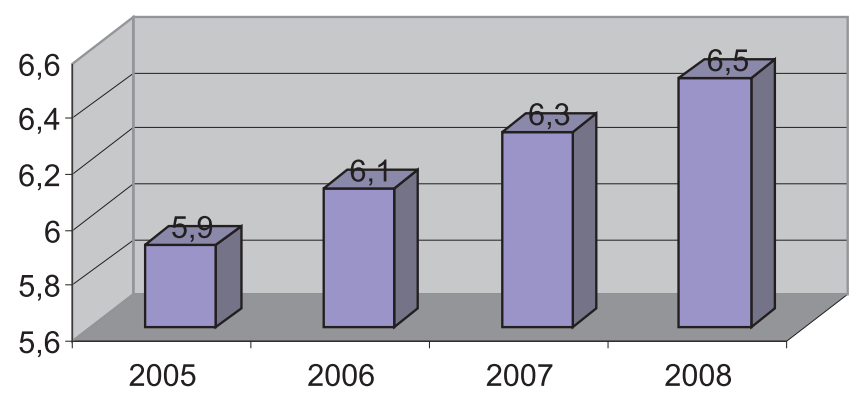

Fuente: Área de Gobierno de Seguridad y Movilidad (2005-2008)

Pero la policía madrileña no sólo recaba datos sobre seguridad objetiva, sino que también es una de sus preocupaciones la percepción de los ciudadanos sobre la seguridad. Así, en relación con la seguridad subjetiva, ésta mejora también, tanto en relación con el conjunto de la ciudad como con su distrito (ver figura 7), lo que constituye una de las tasas de percepción de inseguridad más bajas de todas las capitales europeas.

\section{Figura 7. Grado de seguridad percibida}

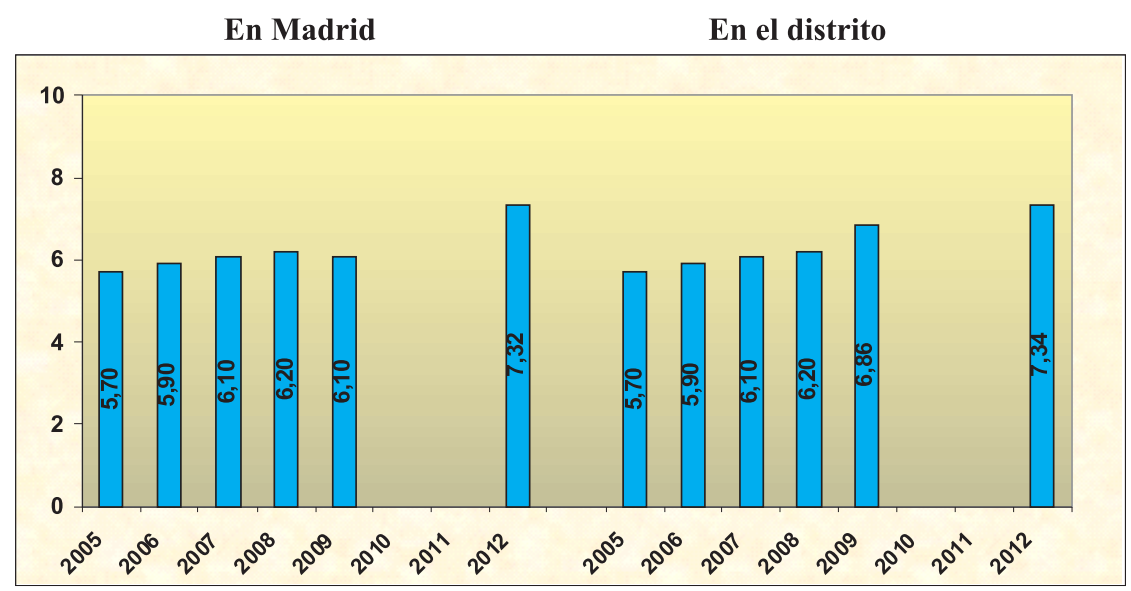

Fuente: Área de Gobierno de Seguridad y Movilidad (2005-2012) 


\section{Conclusiones}

La transformación de la policía municipal de Madrid tiene su origen y motor fundamental en el cambio político de 2003 en la ciudad de Madrid y recibe su impulso del liderazgo del equipo de gobierno y cargos directivos que proceden del anterior gobierno de la Comunidad de Madrid. El proceso de reforma contiene múltiples vectores de actuación que implican:

- la reestructuración orgánica del Área de Gobierno de Seguridad y Movilidad, dividida en dos Coordinaciones situadas bajo la misma dirección política;

- la introducción de profundos cambios en el modelo de gestión, basado en la atención al ciudadano y en la orientación a los resultados, la incorporación de la filosofía de los modelos de gestión de la calidad, la separación de las funciones operativas, administrativas y gerenciales, la aplicación de la gestión por competencias y la dirección por objetivos y el empleo de la planificación estratégica como herramienta.

- La fuerte inversión en medios materiales para dotar a la policía de las infraestructuras tecnológicamente más avanzadas para la prestación del servicio;

- El fuerte aumento de la plantilla de la policía y de su dedicación prioritaria a tareas de seguridad mediante la creación del cuerpo de agentes de movilidad y la externalización de funciones de poco valor añadido;

- La generación de acuerdos de colaboración y de alianzas con los principales actores de la seguridad, sean éstos órganos pertenecientes a la misma o a otra administración, o elementos del tejido asociativo ciudadano; y sobre todo:

- El cambio ideológico sobre la significación de la seguridad, que pasa a ser concebida como un «servicio público» que va mucho más allá del puro ejercicio monopólico y excluyente de la autoridad administrativa, y que hace que la organización en su conjunto tenga como ambicioso objetivo pasar de la prevención del delito a la «prevención del riesgo» mediante el control del ciclo completo de la seguridad desde la prevención y la intervención hasta la investigación.

A pesar de estos logros, la experiencia de profunda transformación organizativa para la configuración de la policía municipal como policía «integral» ha tenido que sortear numerosos obstáculos. En ocasiones, la desconfianza dentro y fuera de la organización; en otros casos, la defensa de privilegios adquiridos y consolidados por el tiempo y la desidia política; las más de las veces, la resignación, la conformidad o la aversión al riesgo. En todo caso, las recientes movilizaciones de los sindicatos gremiales de la policía contra la introducción de elementos salariales condicionados al incremento de la productividad nos recuerdan que la transformación de las organizaciones públicas es compleja, lenta y penosa, y que ningún avance es necesariamente permanente e irreversible. 


\section{Bibliografía}

Arenilla, M. (2008). «La política de modernización al servicio del ciudadano», en El Derecho Público de la Comunidad de Madrid, IMAP, Madrid.

Arenilla, M, (ed.) (2011). Crisis y reforma de la Administración Pública, Netbiblo, Madrid.

Barnejee, T. y Loukaitou-Sideris, A. (2013). «Suspicion, Surveillance and Safety: a new imperative for public space», en Carmon, N. y Fainstein, S.S. Policy, Planning and People, University of Pennsylvania Press, Filadelfia.

Bourgon, J., (2007). «Gobierno receptivo, responsable y respetado. Hacia una «nueva» teoría de la Administración pública», en Revista Internacional de Ciencias Administrativas, marzo, $\mathrm{n}^{\mathrm{o}}$ 1, vol. 73, pp. 5-26.

Cook, P.J. (2009). «Crime in the city», en Inmann, R.I. (ed.): Making cities work, Princeton University Press, New Jersey.

Crespo, J y Pastor, G. (coords.) (2002). Administraciones públicas españolas. McGraw Hill, Madrid.

Crespo, J y Criado, I. (2005). «Evaluando el impacto de la implantación de de la calidad en las Administraciones Públicas. Las Cartas de Servicios en la Comunidad de Madrid», en Revista de Estudios de la Administración Local y Autonómica, n ${ }^{\circ}$ 298-299.

De Bruijn, H. (2002). Managing performance in the public sector. Routledge, Londres.

Eck, John E. et al. (2005). Mapping crime: understanding hot spots, National Institute of Justice, Washington D.C.

Euchner, Ch.C. y McGovern, S.J. (2003). Urban Policy reconsidered, Routledge, Londres.

García Solana, $\mathrm{M}^{\mathrm{a}}$ (20J.01). «La posición del ciudadano: la noción del cliente», en Olías de Lima, Blanca (coord.). La Nueva Gestión Pública, Prentice Hall, Madrid.

Greneron, G. (2010). «Police nationale versus police municipale: mythe ou réalité?», en Pauliat, Hélène (comp.). La sécurité intérieur en Europe: entre protection des citoyens et frénésie sécuritaire?, PULIM, Limoges.

Hill, M. y Hupe, P. (2002). Implementing public policy, Sage, Londres.

Holmberg, L. (2002). «Ersonalized policing : results from a series of experiments with proximity policing in Denmark», en Policing: an International Journal of Police Strategies and Management, $\mathrm{n}^{\mathrm{o}} 1$, vol. 25.

Kelling, G. L. y Wilson, J. Q. (1982). «Broken windows», en The Atlantic, $\mathrm{n}^{\circ}$ 93, vol. 249.

Knoepfel, P., Larrue, C y Varone, F. (2001). Analyse et pilotage des politiques publiques, Helbing \& Lichtenhahn, Ginebra.

Maguire, M. y John, T. (2006). «Intelligence-led policing, managerialism and community engagement: Competing priorities and the role of the National Model in the UK», Policing and Society, $\mathrm{n}^{\mathrm{o}}$ 1, vol. 16.

Martin, E. (2010). «La coexistence des polices nationale et locale: lisibilité et coordination sont nécessaires», en Pauliat, Hélène (comp.): La sécurité intérieur en Europe: entre protection des citoyens et frénésie sécuritaire? PULIM, Limoges.

Naciones Unidas (2011). Introductory Handbook on Policing Urban Space, Naciones Unidas, Programa UN-Habitat, Nueva York.

Olías de Lima, B. (coord.) (2001). La Nueva Gestión Pública, Prentice Hall, Madrid.

Osborne, S y Brown, K. (2005). Managing change and innovation in public service organizations, Routledge, Londres.

Renier, J. L. (2010). «Les polices nationales et locales en Europe», en Pauliat, Hélène (comp.): La sécurité intérieur en Europe: Entre protection des citoyens et frénésie sécuritaire?, PULIM, Limoges. 
Ruano, J. M. (2004). «La transformation des grandes villes espagnoles», en Pauliat, Hélène (comp.): L'autonomie des collectivités territoriales en Europe: Une source potentielle de conflits?, PULIM, Limoges.

Sampson, R. J. y Raudenbush, S. W. (1999). «Systematic social observation of public spaces: A new look at disorder in urban neighborhoods», en American Journal of Sociology, vol. $105, n^{\circ} 3$.

Shamsul, M. (2006). «La Modernización del Gobierno: un Simposio. La Modernización del Gobierno: contribución y crítica», en Revista Internacional de Ciencias Administrativas, $\mathrm{n}^{\circ}$ 72, vol. 3, pp. 337-344.

Sousa, W. H. y Kelling, G. L. (2006). «Of 'broken windows', criminology and criminal justice», en Weisburg, David y Braga, Anthony (eds.). Police innovation: contrasting perspectives, Cambridge University Press, New York.

Tireloque, P. (2010). «Les réponses institutionnelles: la coéxistence délicate entre policess nationales et municipales», en Pauliat, H. (comp.), La sécurité intérieur en Europe: entre protection des citoyens et frénésie sécuritaire?, PULIM, Limoges. 